\title{
Nutritional modulation of gut inflammation
}

\author{
Brian J. Rowlands ${ }^{1 *}$ and Keith R. Gardiner ${ }^{2}$ \\ ${ }^{1}$ Queens Medical Centre and University of Nottingham, Nottingham NG7 2UH, UK \\ ${ }^{2}$ Queens University of Belfast, Belfast BTi2 6BJ, UK
}

In the past 25 years, numerous scientific studies have documented the value of nutrient support in critically-ill patients. Sepsis, systemic inflammatory response syndrome (SIRS) and multiple organ dysfunction syndrome (MODS) complicate several surgical diseases and their treatment by surgical, endoscopic, medical and radiological techniques. Many patients who are treated for sepsis receive either intravenous or enteral nutritional support. Administration of these regimens is governed by a sound understanding of the metabolic response to trauma and basic nutritional principles. Strict protocols for administration minimize the technical, mechanical, metabolic, biochemical, haematological and septic complications. Several diseases have a high incidence of septic complications, SIRS and MODS (McCrory \& Rowlands, 1993). These include major trauma, obstructive jaundice, inflammatory bowel disease (IBD), acute pancreatitis and major intra-abdominal sepsis. These clinical conditions and their septic complications are characterized by a state of 'hypermetabolism', which leads to a rapid consumption of endogenous stores of protein and energy, immunological dysfunction and deterioration of organ function (Cerra, 1987). These changes, which affect the liver, kidney, gastrointestinal tract, heart and lungs, are orchestrated by a series of neuroendocrine events and the release of cytokines, activators and mediators. The 'gut-liver axis' appears to have a central role in these responses. Altered gastrointestinal structure and function and associated changes in hepatic Kupffer cell function contribute to metabolic dysfunction. Parenteral and enteral nutrient substrates as well as pharmacological and immunological therapies may modify these changes (Cerra, 1991; Wilmore, 1991).

In recent years, there have been two major trends in the use of nutritional support. First, there is a major shift from intravenous administration of nutrients to enteral feeding, which is supported by evidence from controlled clinical trials (Kudsk et al. 1992; Moore et al. 1992). Second, the quantity of nutrients being administered is decreasing and the quality of the nutrient mix is improving. Enteral feeding may be delivered using normal oral nutrition, standard enteral diets and supplementary enteral nutrition with novel substrates. Substrates suggested as capable of enhancing intestinal integrity and supporting immune function include amino acids (glutamine, arginine and ornithine), fatty acids (shortchain and $n-3$ polyunsaturated) and nucleotides (RNA; Daly et al. 1992; Carver \& Walker, 1995; Gardiner et al. 1995c).
Enteral nutrients produce their beneficial effects in several ways. They support generalized immune function, enhance mucosal barrier function and reduce bacterial translocation. In addition, they may modify hepatic Kupffer cell function, cytokine release and acute-phase protein production. Enteral diets maintain the structural and functional integrity of the small and large bowel by stimulation of motility, mucus production and gastrointestinal hormone release. Maintenance of the lumen milieu of nutrients, micro-organisms and trophic factors is important for normal digestive and barrier function (Silk \& Grimble, 1994).

The present paper discusses the experimental evidence of gut mucosal barrier dysfunction in the development of sepsis, SIRS and MODS. IBD can lead to disruption of the gastrointestinal mucosal barrier. Experimental and clinical studies of gut inflammation can provide valuable insights into the pathophysiology of sepsis, SIRS and MODS. A major therapeutic strategy for the prevention and treatment of gut-derived sepsis is the provision of enteral nutrition. Individual speciality nutrients (novel substrates) may have a selective action on the maintenance of gut mucosal integrity (selective gut nutrients) or may support general immune function (immuno-modularity nutrients; Gardiner et al. 1995c). The use of these speciality nutrients in critically-ill patients may have beneficial effects on nutritional, metabolic and immune status. This may lead to reductions in complications, length of hospital stay and mortality, but carefullyconducted controlled clinical trials will be necessary to identify those groups of patients most likely to benefit from nutritional interventions (Souba, 1997).

\section{Sepsis, systemic inflammatory response syndrome and multiple organ dysfunction syndrome}

Sepsis and related conditions present a gradation of severity of illness. At one end of the spectrum is minimal derangement of normal physiology and rapid restoration of metabolic homeostasis with therapy. At the other extreme is massive disruption of normal organ function, which leads to progressive deterioration and death despite treatment. Central to our understanding of these events is an appreciation that initially there is a localized response to injury. This may lead subsequently to systemic manifestations if the local host defence mechanisms are inadequate or overwhelmed. Localized infections due to bacteria, viruses, fungi and parasites

\footnotetext{
Abbreviations: IBD, inflammatory bowel disease; MODS, multiple organ dysfunction syndrome; SIRS, systemic inflammatory response syndrome; TNBS, 2,4,6-trinitrobenzene sulfonic acid; TNF, tumour necrosis factor.

*Corresponding author: Professor B. J. Rowlands, fax +44 (0)115970 9428, email bjr.surgery@nottingham.ac.uk
} 
stimulate the release of various mediators, e.g. cytokines, prostaglandins, thromboxanes, platelet-activating factors and the complement system. These help to combat infection by activating neutrophils, with consequent degranulation and release of oxygen radicals which increase local blood flow and vascular permeability allowing the influx of phagocytic cells. They also activate leucocytes and induce chemotaxis. If the severity of the infection is sufficient that these mediators spill over into the systemic circulation, a septic cascade is initiated which may lead to septic shock, SIRS, and MODS (Davies \& Hagen, 1997). Superoxide radicals now damage host cells. Endotoxin and various cytokines, activators and mediators, e.g. tumour necrosis factor (TNF), the interleukins, transforming growth factor $\beta$ and prostaglandin $E_{2}$ all contribute to the initiation and maintenance of this cascade, which may be beneficial or detrimental, depending on the clinical setting.

Sepsis is not synonymous with overwhelming infection, and in many patients who fit the criteria for SIRS, no microorganism can be demonstrated or cultured (Rowlands, 1997). Our understanding of the development of organ failure and death has expanded greatly since originally described by Baue (1975). We now recognize three stages in the development of SIRS (Bone, 1996a). The balance between the antiinflammatory systemic response and the pro-inflammatory systemic response is important for metabolic homeostasis, and the disruption of this equilibrium gives rise to a number of syndromes (compensatory anti-inflammatory syndrome, mixed antagonist response syndrome, MODS; see Fig. 1). Their clinical manifestations are cardiovascular compromise (shock), suppression of immunity, apoptosis and organ dysfunction (Bone, 1996b; Nieuwenhuijzen et al. 1996). The importance of cytokines, their natural antagonists and endogenous antibodies to endotoxins in determining outcome in patients with sepsis syndrome has been demonstrated by Goldie et al. (1995). This study provides an explanation of why most new immunological therapies have failed to produce clinical improvement in septic patients (Rowlands, 1997).

\section{Gut barrier function in health}

The gastrointestinal tract is usually thought of primarily as an organ of digestion and absorption, but it is a metabolicallyactive organ that requires specific nutrients (Page, 1989). It has a major barrier function, protecting the body from harmful intraluminal pathogens and large antigenic molecules (Saadia et al. 1990). In addition, it plays a pivotal role in the metabolism of glutamine (Souba et al. 1985). The gut mucosal barrier comprises both immunological and nonimmunological protective components, the former being divided into local and systemic components and the latter comprises mechanical and chemical barriers as well as intraluminal bacteria (Table 1). The maintenance of normal epithelial cell structure prevents trans-epithelial migration of particles from the gut lumen, and the preservation of tight junctions between the cells prevents movement through the para-cellular channels (van Leeuwen et al. 1994). Acid secretion in the stomach, alkali secretions in the small bowel and mucus production throughout the gastrointestinal tract

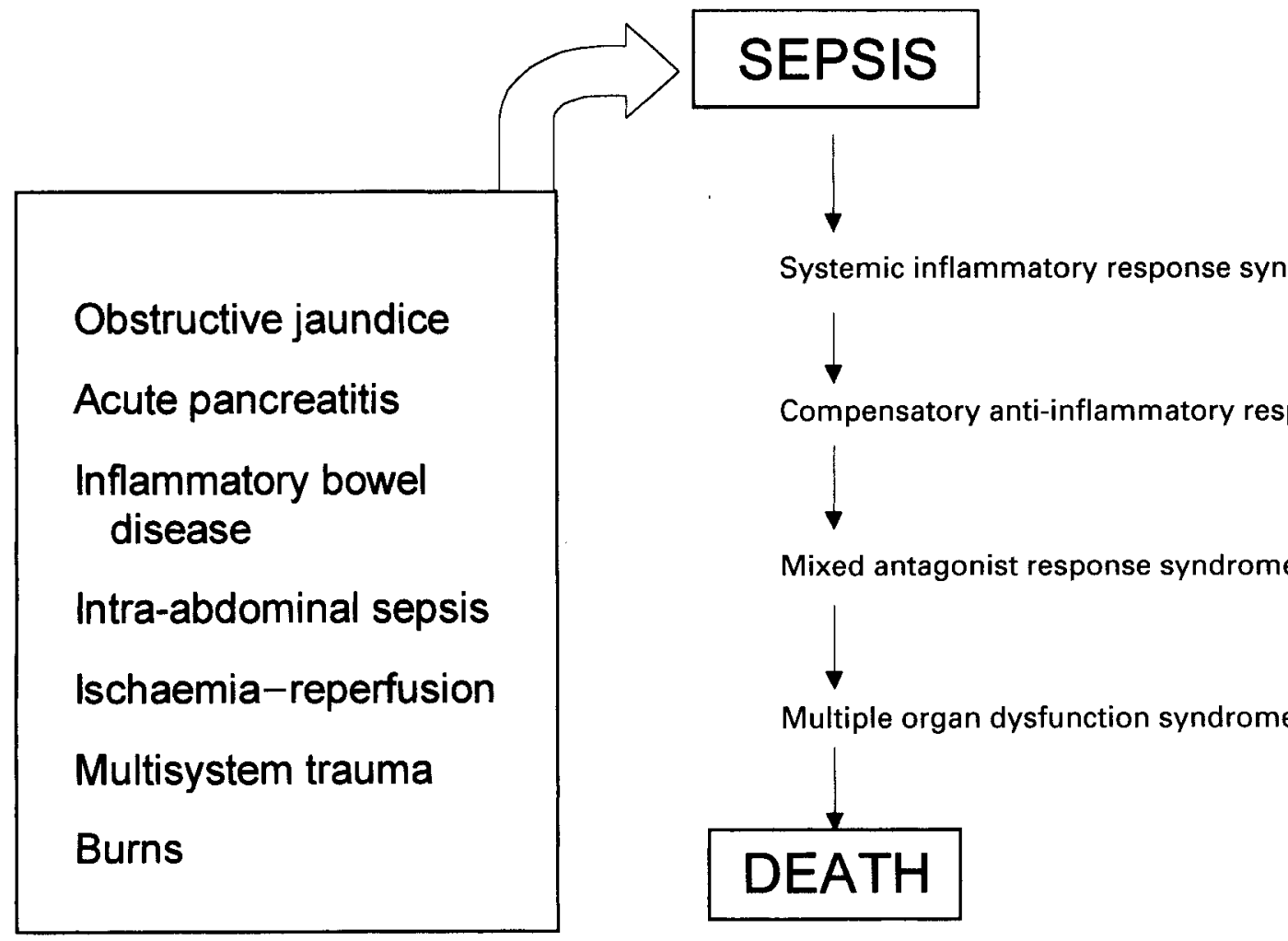

Fig. 1. A number of common surgical conditions have a high incidence of septic complications, which lead to the development of a number of well-recognized syndromes and death. 
provides additional protection. The lumen of the gut is colonized by aerobic and anaerobic micro-organisms, and there is a progressive increase in their numbers from the stomach, where gastric acid produces an almost sterile environment, to the colon, which harbours $10^{8}$ aerobes and $10^{11}$ anaerobes. Under normal circumstances, these micro-organisms remain within the lumen of the bowel where they have important functions in metabolic and nutritional homeostasis. In disease states, when the mucosal barrier is compromised, these micro-organisms and their toxic products may 'escape' from the lumen to produce deleterious systemic effects (Fig. 2).

Table 1. Components of the gut mucosal barrier

\begin{tabular}{ll}
\hline Immunological & Non-immunological \\
\hline Local & Mechanical \\
Gut-associated lymphoid tissue & Healthy enterocyte \\
Intra-epithelial lymphocytes & Tight junction \\
Submucosal aggregates & Cell turnover \\
Peyer's patches & Normal motility \\
Mesenteric lymph nodes & Chemical \\
Secretory lgA & Gastric acidity \\
Systemic & Salivary lysozyme \\
Circulatory lymphocytes & Lactoferrin \\
Hepatic Kupffer cells & Mucus secretion \\
& Bile salts \\
& Bacteriological \\
& Aerobic micro-organisms \\
& Anaerobic micro-organisms \\
\hline
\end{tabular}

$\lg \mathbf{A}$, immunoglobulin $\mathbf{A}$.
Gut barrier function in disease

The demonstration of intestinal atrophy manifested by changes in weight, structure and mucosal content of DNA and protein has been assumed to indicate impaired intestinal barrier function, but this has not been confirmed in animal studies of protein malnutrition (Deitch, 1990). Recent clinical evidence demonstrates a strong association between compromise of gut barrier function and malnutrition, which suggests a mechanism that facilitates gut-derived infection and sepsis (Reynolds et al. 1996; Welsh et al. 1998). Dysfunction of the mucosal barrier is thought to result from an imbalance of aggressive and defensive factors on the gastrointestinal mucosa (Sartor, 1990). Genetic and environmental factors may modify the response of the gastrointestinal mucosa to pro-inflammatory factors or may modify the protective factors. Increased gastric acid production, colonization with Helicobacter pylori, ingestion of non-steroidal anti-inflammatory analgesics and the use of broad-spectrum antibiotics, predispose the gastrointestinal mucosa to ulceration. Maintenance of mucosal blood flow (Fink, 1991), oxidative fuel supply (Alverdy et al. 1988) and a normal intestinal bacterial flora (Barber et al. 1991) protect against barrier dysfunction. Failure of the intestinal mucosal barrier results in permeation of microbial and dietary antigens across the intestinal wall. The trans-mural migration of enteric bacteria or their products (endotoxins) to extra-intestinal sites has been termed translocation, and may occur by the transcellular or para-cellular routes. Enhanced uptake of macromolecules and bacteria has been demonstrated where the intestinal mucosa is damaged by inflammation, infection,

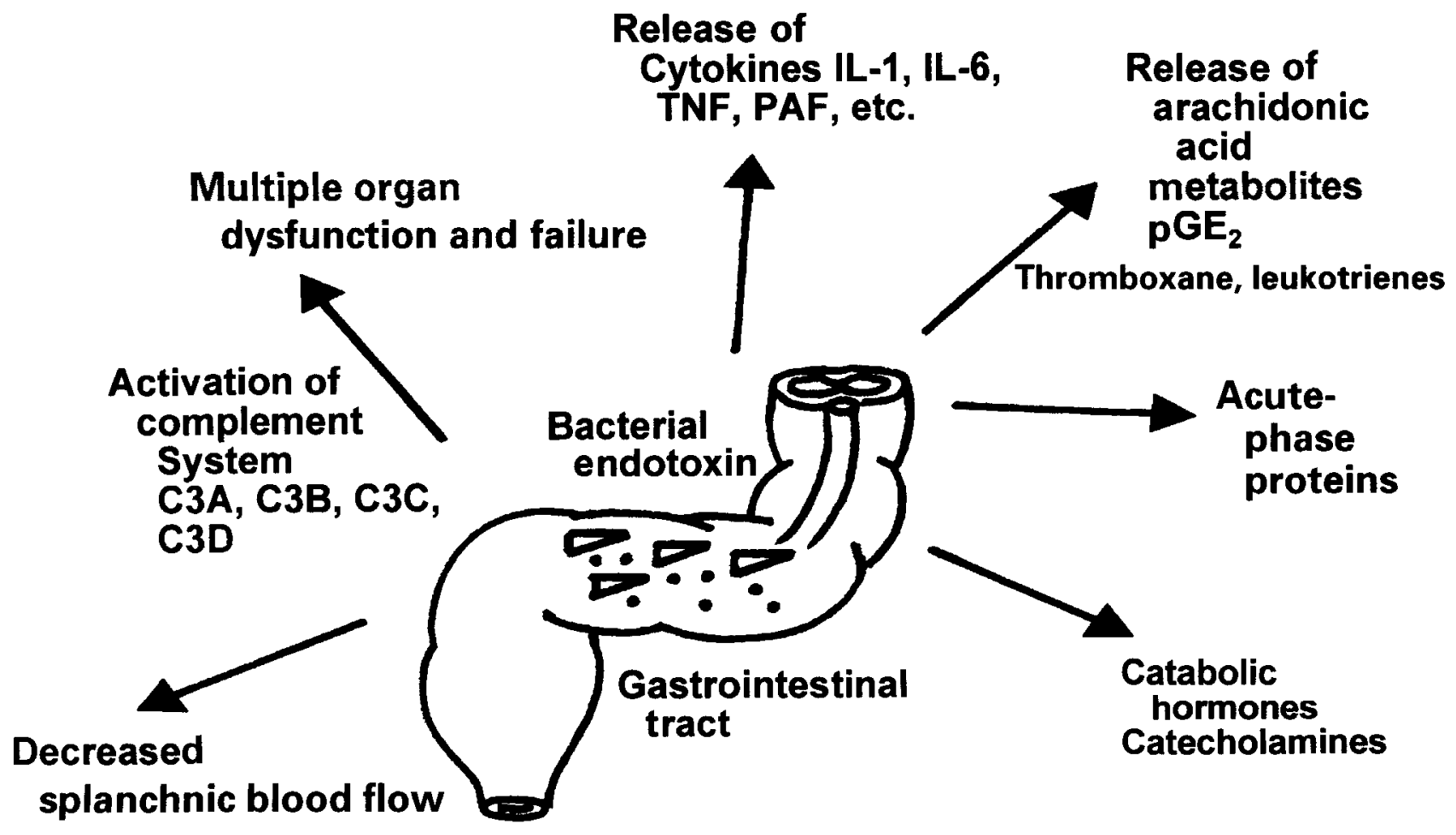

Fig. 2. Various diseases lead to compromise of gut mucosal barrier function. Breakdown of local defences allows translocation of bacteria and toxin. In turn, they activate a number of systemic inflammatory cascades and release of mediators, cytokines, hormones and acute-phase proteins, which further compromise host defences. C3A-D, components of the complement system; IL, interleukin; PAF, platelet-activating factor; $P G$, prostaglandin; TNF, tumour necrosis factor. 
neoplasia or trauma. In addition, systemic endotoxaemia, bacterial translocation, and increased intestinal permeability to macromolecules have been demonstrated in patients with rheumatic diseases, haemorrhagic shock, burns, burn sepsis, major trauma, following chemotherapy or radiotherapy, and in experimental endotoxaemia in the absence of macroscopic intestinal disease. However, translocation of endotoxin or bacteria has not been found commonly in patients after major trauma or burns (Gardiner et al. 1995c).

The clinical significance of intestinal mucosal barrier dysfunction is more difficult to determine. Clinical studies strongly suggest that intestinal barrier dysfunction and increased permeability occur in patients with intestinal inflammation and other diseases associated with increased mortality and morbidity. It is hypothesized that translocating micro-organisms and toxins activate a systemic inflammatory cascade and promote organ dysfunction and failure, but it is difficult to demonstrate a causal link (Cerra, 1991).

\section{Gut barrier dysfunction in inflammatory bowel disease}

Patients with IBD may exhibit a number of manifestations of malnutrition. They often present with a history of anorexia, nausea, vomiting and abdominal pain, symptoms which are associated with poor nutritional intake. Diarrhoea or highoutput fistulas or stomas may lead to significant nutrient losses. There may also be evidence of malabsorption of vitamins, minerals, bile salts, and steatorrhoea with protein loss. Acute depletion of tissue stores of endogenous energy and protein stores occurs rapidly, leading to clinical malnutrition, poor wound healing, weight loss and immune dysfunction. Gut mucosal barrier dysfunction contributes significantly to this state of increased metabolism.
In the Department of Surgery at Queen's University of Belfast, we investigated gut barrier dysfunction in IBD by measuring systemic endotoxin concentrations in patients with quiescent and active disease. In IBD patients requiring hospitalization for acute relapse, systemic endotoxaemia was demonstrated in $88 \%$ of patients with ulcerative colitis ( $n$ 25), $75 \%$ with indeterminate colitis $(n 8)$ and $94 \%$ of those with Crohn's disease ( $n$ 31). In a group of outpatients with inactive disease, systemic endotoxaemia was demonstrated in $33 \%$ of patients with ulcerative colitis ( $n 33)$ and in $9 \%$ of those with Crohn's disease (n 34; Gardiner et al. $1995 \mathrm{~b}$ ). The clinical significance of gut barrier dysfunction in IBD was examined by seeking a correlation between systemic endotoxaemia and disease extent or activity, and by searching for evidence of a specific immune response (production of anti-endotoxin antibodies or the cytokine TNF). Systemic endotoxaemia was found to correlate positively with anatomical extent and clinical activity of ulcerative colitis. Circulating TNF was detected in $40 \%$ of patients with ulcerative colitis and $45 \%$ with Crohn's disease. Plasma TNF and soluble TNF receptor p55 concentrations correlated positively with clinical and laboratory measures of disease activity (Gardiner et al. 1995b). In patients with Crohn's disease, there were significant increases in the plasma concentrations of antibodies (immunoglobulin G) to the endotoxin core which were found to correlate positively with systemic endotoxaemia. These findings support a central role for endotoxin in the pathogenesis of IBD (Fig. 3).

The pathogenesis of colonic mucosal barrier dysfunction was investigated in an experimental model of colitis using intraluminal instillation of 2,4,6-trinitrobenzene sulfonic acid (TNBS) and ethanol (Morris et al. 1989). This model has been well characterized and possesses clinical

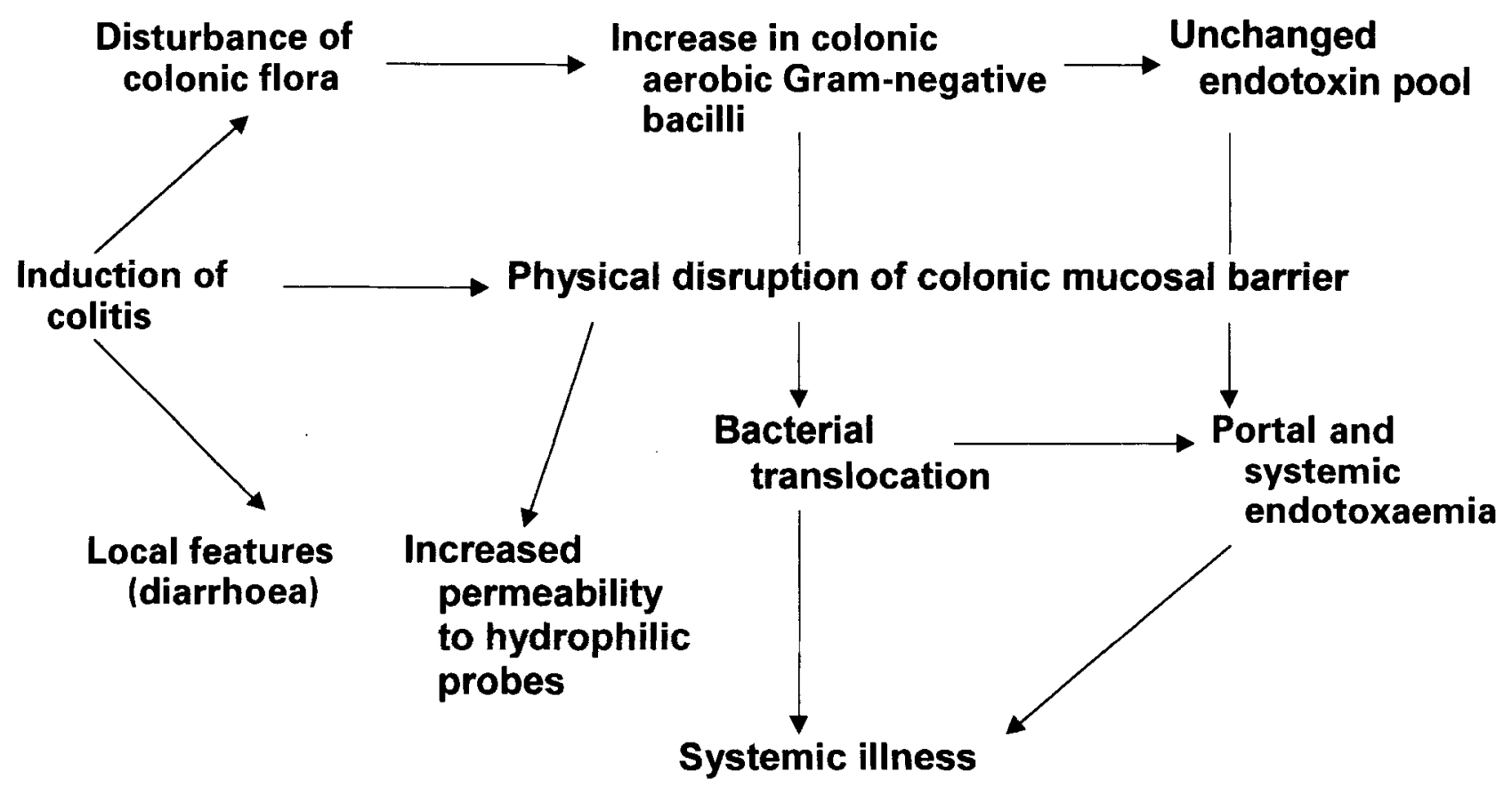

Fig. 3. The pathogenesis of inflammatory bowel disease demonstrating the central role for endotoxin in the development of systemic illness. 
biochemical and pathological similarities to colonic Crohn's disease. In this TNBS model, systemic endotoxaemia correlates positively with the macroscopic appearance of the colitis and with serum concentrations of lactate and $\alpha_{2}$-macroglobulin (Gardiner et al. 1995a; Neilly et al. $1995 a$ ). There was an initial reduction in both immunoglobulin $\mathrm{G}$ and $\mathrm{M}$ anti-endotoxin core antibody, with a subsequent increase. This may represent a consumption of antibody by translocating endotoxin, followed by a B-cell response and an increase in antibody production. Increased colonic permeability to the hydrophilic probe polyethylene glycol (molecular mass 4000) and increase in the translocation of colonic bacteria correlate positively with severity of colitis (Gardiner et al. 1995a). A significant increase in the numbers of colonic Gram-negative bacilli and a decrease in the Gram-positive aerobic bacilli count occurred, but there was no effect on the faecal endotoxin load. There was a positive correlation between the severity of colonic inflammation and the extent of bacterial translocation in colitic animals (Gardiner $e$ t al. 1993). Disruption of the physical barrier is more important than overgrowth of Gram-negative bacilli or release of endotoxin in promoting translocation in experimental colitis.

\section{Treatment of gut mucosal barrier dysfunction}

Support of the gut mucosal barrier includes adequate mucosal perfusion, optimal $\mathrm{O}_{2}$ delivery, prevention of gastrointestinal haemorrhage and maintenance of lumen micro-ecology. These general measures, together with more specific measures, support enterocyte structure and function. An important component of support is enteral nutritional therapy. There is convincing evidence that administration of enteral nutrition when compared with parenteral feeding is associated with improved immune function and reduced septic complications following major trauma. Other treatments that may contribute to resolution are the reduction of the intestinal pool of Gram-negative bacteria and endotoxin, enhancement of mucosal healing, supporting general immune function and neutralizing the systemic effects of bacteria and their toxins (Gardiner et al. 1995c).

In IBD, gut barrier dysfunction improves when the intestinal pool of Gram-negative bacteria and endotoxin is reduced by the administration of non-selective or selective antibiotics, of lactulose to promote non-pathogenic lactobacilli, or of adsorbents to bind intraluminal endotoxin. Treatment may be directed at enhancing healing of the intestinal mucosa by administration of anti-inflammatory drugs (steroids) systemically or topically (Neilly et al. 1995b). Treatment may be directed at removing or neutralizing extra-intestinal bacteria endotoxin or other cytokines, activators or mediators. Intravenous injection of taurolidine, a drug with antiseptic, antibiotic and anti-endotoxin activity, significantly reduced systemic endotoxaemia in TNBS colitis, whereas systemic broad-spectrum antibiotic (metronidazole and cefuroxime) therapy was ineffective (Gardiner et al. 1994). Administration of anti-TNF antibody (cTN3) significantly reduced systemic endotoxaemia, plasma interleukin-6 concentration, acute-phase protein response and weight loss in TNBS colitis. Repair of the intestinal barrier may be hastened by multi-targeted therapy aimed at reducing luminal aggressive factors, reducing the inflammatory response (steroids, nitric oxide synthase inhibition, anti-cytokines) and selective gut nutrition.

The role of novel substrates in maintaining gut integrity has been extensively reviewed by considering their metabolism in health and disease, the effects on the gut mucosa and the experimental and clinical evidence supporting their use in clinical practice (Gardiner et al. 1995c). Evidence that these substrates improve gut mucosal barrier function and increase survival does not necessarily imply that restored barrier function and improved survival are causally linked. The substrates may exert their beneficial effects by improving $\mathrm{N}$ balance and metabolism, or by enhancing immune function and clearance of translocated bacteria. From experimental and clinical studies to date the strongest candidates as selective gut nutrients are glutamine for the enterocyte and short-chain fatty acids for the colonocyte. Recent evidence shows that glutamine-supplemented parenteral nutrition improves outcome in critically-ill intensive care unit patients and surgical patients (Griffiths et al. 1997; Morlion et al. 1998). The results from studies of enteral supplementation of glutamine are disappointing (Powell-Tuck, 1997), and quite modest intakes of standard oral diet $(0.6 \mathrm{~g} \mathrm{~N} / \mathrm{kg}$ daily) are sufficient to maintain gut integrity and immune function. Short-chain fatty acids and $n-3$ polyunsaturated fatty acids have modest beneficial effects in patients with intestinal inflammation (Ross, 1993). Arginine has proven immunological benefits (Barbul, 1986). Despite this finding, oral supplementation with arginine has been shown to be detrimental in the TNBS-induced colitis model, causing increased colonic inflammation (Neilly et al. 1995c), but beneficial to mucosal barrier function and survival after experimental ischaemiareperfusion injury (Schleiffer \& Raul, 1996). There is growing evidence that the L-arginine-nitric oxide pathway is important in the development of colonic inflammation, and that this may lead to new therapeutic strategies in IBD (Kimura et al. 1998; Warner \& McCartney, 1998). Arginine supplements for critically-ill patients should be used cautiously at present, and there is insufficient evidence from clinical studies to support the use of orthinine, branchedchain amino acid or nucleotide supplementation by themselves. However, there is some evidence that supplementation of enteral diets with combinations of novel substrates is beneficial to patients in a number of different clinical situations where gut barrier function is compromised by malnutrition and hypermetabolism (Bower et al. 1995; Daly et al. 1995). The use of diets (containing fibre, fermented oats and lactobacillus) that support probiotic bacteria (microbial interference treatment) has been advocated as an important new development in the support of these patients (Bengmark, 1996, 1998).

\section{Summary}

Nutritional therapy is an important component of the management of gastrointestinal inflammation, which disrupts the gut mucosal barrier leading to sepsis, SIRS and MODS. Future studies will be needed to define the role of specific nutrients in enhancing mucosal barrier function and supporting general immune function, and how this affects morbidity and mortality of critically-ill patients. 


\section{References}

Alverdy J, Aoys E \& Moss G (1988) Total parenteral nutrition promotes bacterial translocation from the gut. Surgery 104, 185-190.

Barber AE, Jones WG, Minei JP, Fahey TJ, Lowry SF \& Shires GT (1991) Bacterial overgrowth and intestinal atrophy in the etiology of gut barrier failure in the rat. American Journal of Surgery 161, 300-304.

Barbul A (1986) Arginine: biochemical, physiology and therapeutic implications. Journal of Parenteral and Enteral Nutrition 10, 227-238.

Baue AE (1975) Multiple progressive or sequential systems failure. Archives of Surgery 110, 779-781.

Bengmark S (1996) Econutrition and health maintenance - a new concept to prevent GI inflammation, ulceration and sepsis. Clinical Nutrition 15, 1-10.

Bengmark S (1998) Ecological control of the gastrointestinal tract the role of probiotic flora. Gut $\mathbf{4 2}, 2-7$.

Bone RC (1996a) Toward a theory regarding the pathogenesis of the systemic inflammatory response system - what we do and do not know about cytokine regulation. Critical Care Medicine 24, 163-172.

Bone RC (1996b) Sir Isaac Newton, sepsis, SIRS and CARS. Critical Care Medicine 24, 1125-1128.

Bower RH, Cerra FB, Bershadsky B, LiCari J, Hoyt D, Jensen GL, van Buren CT, Rothkopf MM, Daly JM \& Adelsberg BR (1995) Early enteral administration of formula (Impact) supplemented with arginine, nucleotides and fish oil in intensive care unit patients: results of a multicenter prospective, randomized clinical trial. Critical Care Medicine 23, 436-449.

Carver JD \& Walker WA (1995) The role of nucleotides in human nutrition. Nutritional Biochemistry 6, 58-72.

Cerra FB (1987) Hypermetabolism: organ failure and metabolic support. Surgery 101, 1-14.

Cerra FB (1991) Nutrient modulation of inflammatory and immune function. American Journal of Surgery 161, 230-234.

Daly JM, Leibermann MD, Goldfine J, Shou J, Weintraub F, Rosato EF \& Lavin P (1992) Enteral nutrition with supplemental arginine, RNA and omega-3-fatty acids in patients after operation: immunological, metabolism and clinical outcome. Surgery 112, 56-67.

Daly JM, Weintraub FN, Shou J, Rosato EF \& Lucia M (1995) Enteral nutrition during multimodality therapy in upper gastrointestinal cancer patients. Annals of Surgery 221, 327-338.

Davies MG \& Hagen P-O (1997) Systemic inflammatory response syndrome. British Journal of Surgery 84, 920-935.

Deitch EA (1990) Bacterial translocation of the gut flora. Journal of Trauma 30, S184-S189.

Fink MP (1991) Gastrointestinal mucosal injury in experimental models of shock trauma and sepsis. Critical Care Medicine 19, $627-641$.

Gardiner KR, Anderson NH, McCaigue MD, Erwin PJ, Halliday MI \& Rowlands BJ (1994) Enteral and parenteral antiendotoxin treatment in experimental colitis. Hepato-Gastroenterology 41, 554-558.

Gardiner KR, Anderson NH, Rowlands BJ \& Barbul A (1995a) Colitis and colonic mucosal barrier dysfunction. Gut 37, $530-535$.

Gardiner KR, Erwin PJ, Anderson NH, Barr JG, Halliday MI \& Rowlands BJ (1993) Colonic bacteria and bacterial translocation in experimental colitis. British Journal of Surgery 80, 512-516.

Gardiner KR, Halliday MI, Barclay GR, Milne L, Brown D, Stephens S, Maxwell RJ \& Rowlands BJ (1995b) Significance of systemic endotoxaemia in inflammatory bowel disease. Gut $\mathbf{3 6}$, 897-901.
Gardiner KR, Kirk SJ \& Rowlands BJ (1995c) Novel substrates to maintain gut integrity. Nutrition Research Reviews 8, 43-66.

Goldie AS, Fearon KCH, Ross JA, Barclay GR, Jackson RE, Grant IS, Ramsey G, Blyth AS \& Cameron Howie J (1995) Natural cytokine antagonists and endogenous anti-endotoxin core antibodies in sepsis syndrome. Journal of the American Medical Association 274, 172-177.

Griffiths RD, Jones C \& Allan-Palmer TE (1997) Six month outcome of critically ill patients given glutamine-supplemented parenteral nutrition. Nutrition 13, 295-302.

Kimura H, Hokari R, Miura S, Shigematsu T, Hirokawa M, Akiba Y, Kurose I, Higuchi H, Fujimori H, Tsazuki Y, Serizawa H \& Ishii H (1998) Increased expression of an inducible isoform of nitric oxide synthase and the formation of peroxynitrite in colonic mucosa of patients with active ulcerative colitis. Gut $\mathbf{4 2}$, 180-187.

Kudsk KA, Groce MA, Fabian TC, Minard G, Tolley EA, Poret HA, Kuhl MR \& Brown RO (1992) Enteral versus parenteral feeding: effects of septic morbidity after blunt and penetrating abdominal trauma. Annals of Surgery 215, 503-513.

McCrory DC \& Rowlands BJ (1993) Septic syndrome and multiple system organ failure. Current Practice of Surgery 5, 211-215.

Moore FA, Feliciano DV, Andrassy RJ, McArdle AH, Booth FV, Morganstein-Wagner TB, Kellum JM, Welling RE \& Moore EE (1992) Early enteral feeding, compared with parenteral reduces postoperative septic complications - the results of a metaanalysis. Annals of Surgery 216, 172-183.

Morlion BJ, Stehle P, Wachtler P, Seidhoft H-P, Köller M, König W, Furst P \& Puchstein C (1998) Total parenteral nutrition with glutamine dipeptide after major abdominal surgery: a randomised double blind, controlled study. Annals of Surgery 227, 302-308.

Morris GP, Beck PL, Herridge MS, Depar WT, Szewczuk MR \& Wallace JL (1989) Hapten-induced model of chronic inflammation and ulceration in the rat colon. Gastroenterology $\mathbf{9 6}$, 795-803.

Neilly PJD, Gardiner KR, Kirk SJ, Jennings G, Anderson NH, Elia M \& Rowlands BJ (1995a) Endotoxaemia and cytokine production in experimental inflammatory bowel disease. British Journal of Surgery 82, 1479-1482.

Neilly PJD, Gardiner KR, Kirk SJ, O'Hare M, Jennings G, Anderson NH, Elia M \& Rowlands BJ (1995b) Topical glutamine therapy in experimental inflammatory bowel disease. Clinical Nutrition 14, 283-287.

Neilly PJD, Kirk SJ, Gardiner KR, Anderson NH \& Rowlands BJ (1995c) Manipulation of the L-arginine-nitric oxide pathway in experimental colitis. British Journal of Surgery 82, 1188-1191.

Nieuwenhuijzen GAP, Deitch EA \& Goris RJA (1996) Infection, the gut, and the development of the multiple organ dysfunction syndrome. European Journal of Surgery 162, 259-273.

Page CP (1989) The surgeon and gut maintenance. American Journal of Surgery 158, $485-490$.

Powell-Tuck J (1997) Glutamine supplementation in artificial nutritional support. Lancet $\mathbf{3 5 0}, 534$.

Reynolds JV, O'Farrely C, Feighery C, Murchan P, Leonard N, Fulton G, O'Morain C, Keane FBV \& Tanner WA (1996) Impaired gut barrier function in malnourished patients. British Journal of Surgery 83, 1288-1291.

Ross E (1993) The role of marine fish oils in the treatment of ulcerative colitis. Nutrition Reviews 51, 47-49.

Rowlands BJ (1997) Management of severe sepsis and intensive care. In A Companion to Specialist Surgical Practice - Emergency Surgery and Critical Care, pp. 289-308 [S Patterson-Brown, editor]. London: W.B. Saunders Co. 
Saadia R, Schein M, McFarlane C \& Boffard KD (1990) Gut barrier function and the surgeon. British Journal of Surgery 77, 487-492.

Sartor RB (1990) Role of intestinal microflora in initiation and perpetuation of inflammatory bowel disease. Canadian Journal of Gastroenterology 4, 271-277.

Schleiffer R \& Raul F (1996) Prophylactic administration of L-arginine improves the intestinal barrier function after mesenteric ischaemia. Gut 39, 194-198.

Silk DBA \& Grimble GK (editors) (1994) Gut mucosal nutritional support: enteral nutrition as primary therapy? Gut 35, Suppl. 1, S1-S80.

Souba WW (1997) Nutritional support. New England Journal of Medicine 336, 41-48.
Souba WW, Smith RJ \& Wilmore DW (1985) Glutamine metabolism by the intestinal tract. Journal of Parenteral and Enteral Nutrition 9, 608-617.

van Leeuwen PAM, Boermeester MA, Houdijk APJ, Ferwerda CC, Cuesta MA, Meyer S \& Wesdorp RIC (1994) Clinical significance of translocation. Gut 35, Suppl. 1, S28-S34.

Warner TD \& McCartney SA (1998) NOS inhibitors in colitis: a suitable case for treatment. Gut 42, 152-153.

Welsh FKS, Farmery SM, MacLennan K, Sheridan MB, Barclay GR, Guillou PJ \& Reynold JV (1998) Gut barrier function in malnourished patients. Gut 42, 396-401.

Wilmore DW (1991) Catabolic illness: strategies for enhancing recovery. New England Journal of Medicine 325, 695-702. 